\title{
Economía Circular: ¿Un nuevo paradigma en la economía? Circular Economy: A New Paradigm in Economics?
}

Roger Loyola ${ }^{1}$

${ }^{1}$ Universidad Nacional Agraria La Molina

Correo electrónico: rogerloyola@lamolina.edu.pe

\section{Resumen}

La Economía Circular (EC) aparece como una propuesta novedosa para tener un sistema productivo que pueda ser menos impactante al ambiente. Llama la atención que siendo un concepto llamado economía la participación de los economistas es bien limitada en este campo, por ello se presenta las raíces del mismo y se propone como esta podría ser integrada dentro del análisis económico, justamente haciendo referencia a restricciones adicionales que deberían ser consideradas en este caso. A partir de algunas revisiones bibliográficas se plantean las principales limitaciones que tendrían este tipo de propuestas para implementarse. Finalmente se deja el mensaje que para que la EC funcione esta deba también tener su respectivo análisis económico.

Palabras clave: ciudades de montaña; urbanización; turismo; agricultura; América Latina

\begin{abstract}
The Circular Economy (CE) appears as a novel proposal to have a productive system that can be less impactful to the environment. It is striking that being a concept called economics, the participation of economists is limited in this field, for this reason, its roots are presented and it is proposed how it could be integrated into the economic analysis, precisely by referring to additional restrictions that should be considered in this case. Based on bibliographic reviews, the main limitations that this type of proposal would have to be implemented are raised. Finally, the message is left that for the EC to function, it must also have its respective economic analysis.
\end{abstract}

Keywords: Circular economy, environmental sustainability, Economic rationality 


\section{Introducción}

Las zonas montañosas en todo el mundo están sufriendo cambios respecto al Años después de los principales acuerdos globales sobre la necesidad de proteger el ambiente, especialmente de la reunión de Río $1992^{2}$, los avances sobre las metas ambientales que deben ser alcanzadas son bastante preocupantes. Los niveles de alerta sobre la utilización antropogénica de la naturaleza han generado diversas iniciativas sin aun encontrar una solución acorde a la dimensión del problema. UN Environment (2019) menciona que durante las últimas décadas, las actividades humanas, como el cambio climático causado por el hombre y otros impactos humanos en los ecosistemas, han transformado los sistemas naturales de la Tierra, excediendo su capacidad y alterando sus mecanismos de autorregulación, con consecuencias irreversibles para la humanidad y que esta se ha visto seriamente afectada por los cambios producidos, como el cambio climático y el cambio de uso de la tierra poniendo el peligro los cimientos ecológicos de la sociedad humana y los sistemas naturales que sustentan a otras especies y brindan servicios ecosistémicos invaluables. Igualmente, en UNEP (2021) se menciona que tras una caída sin precedentes del 5,4\% en 2020, las emisiones globales de dióxido de carbono se recuperaron a los niveles anteriores al COVID y las concentraciones de GEI en la atmósfera siguen aumentando. Esto significa que las perspectivas es que los problemas ambientales, y de supervivencia de la especie humana, se agudicen sino se toman las medidas del caso.

En este contexto, hay diversas iniciativas que se han venido implementando tanto a nivel global como a nivel de cada país, desde acuerdos globales hasta implementación de normativas internas que justamente visan con generar medidas que eviten poner en riesgo la sostenibilidad del planeta. En este contexto se han generado diversas propuestas de cómo tener medidas que permitan formas de producción que puedan ser menos agresivas con relación al ambiente. Es así como uno de los conceptos más utilizados en los últimos tiempos es el de Economía Circular (EC) llamando la atención de gobiernos y negocios en particular señalándola como una propuesta innovadora y una forma de poder alcanzar niveles de desarrollo efectivamente sostenibles en la medida que implica que elementos que salen de un proceso sean compartidos por otros y que además de ello nuevos procesos sean menos intensivos en utilización de energía y materiales. Saha et al (2021) señalan que la EC es un concepto ampliamente estudiado en China y la Unión Europea (UE) pero aún está en su etapa inicial en las economías emergentes.

Para Figge et al (2021) la economía circular es una vía hacia la ecoeficiencia, y dado que no se desperdician recursos, la ecoeficiencia alcanza el infinito, y que sin embargo a pesar de su creciente popularidad los sistemas completamente circulares siguen siendo una propuesta puramente conceptual. Sostienen que, en una economía completamente circular, estos recursos se utilizarían un número infinito de veces, por lo que se puede asumir que al menos algunos de los flujos de recursos no son ni completamente lineales ni completamente circulares en la actualidad.

Este interés ha estado presente en la sociedad peruana especialmente en el Ministerio de la Producción y del Ambiente, aprobándose el Decreto Supremo que aprueba la Hoja de Ruta hacia una Economía Circular en el Sector Industria

${ }^{2}$ https://www.un.org/spanish/esa/sustdev/documents/declaracionrio.htm. Declaración de Río sobre el Medio Ambiente y el Desarrollo. 


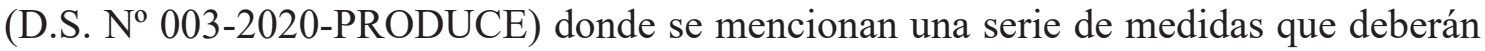
ser llevadas a cabo por diferentes instituciones en acciones de corto, mediano y largo plazo, las mismas que corresponden a 1,3 y 5 años.

En este sentido es importante conceptualizar el significado de economía circular, especialmente desde el punto de vista económico, poder compararlo con otros esfuerzos que ya se han venido haciendo al respecto para a partir de ello evaluar la factibilidad de su implementación especialmente en sociedades donde aún no se ha alcanzado niveles de desarrollo alto en su implementación.

\section{Los antecedentes}

La necesidad de tener un límite para el uso de la naturaleza ya sea como proveedor de insumos, receptor de residuos, así como sistema para la vida, viene desde una larga data. Libros como el de Pearce y Turner ${ }^{3}$ ya mencionaban las reglas que se debían de seguir para tener un sistema sostenible que son: usar recursos no renovables dependiendo de su posibilidad de sustitución por renovables; que ningún recurso renovable deberá utilizarse a un ritmo superior al de su generación; y que las emisiones no deberán ser mayores que la capacidad de carga del ambiente. La idea es que siguiendo estas reglas sería posible mantener la capacidad del ecosistema tierra de soportar las actividades humanas. Es claro que estas reglas no fueron nunca un consenso especialmente porque existían al menos dos formas de ver esta sostenibilidad, la débil y la fuerte. Según Shi et al (2019) la sostenibilidad débil es una visión centrada en el ser humano en la que el capital natural puede ser reemplazado por capital manufacturado dado que según la óptica de la economía del bienestar neoclásica la sostenibilidad débil considera la cantidad total de capital manufacturado y capital natural como la más importante, siendo que lo relevante es el capital total, aun cuando el capital natural se degrade a un estado irrecuperable, este podría ser siendo sostenible. En el caso de la sostenibilidad fuerte señalan que esta una visión centrada en la naturaleza donde el capital natural juega un papel insustituible en la producción y el consumo, siendo que este concepto se basa principalmente en la teoría económica del estado estacionario de que el capital manufacturado no puede duplicarse (aumentarse) sin el aporte de capital natural por lo que el proceso de desarrollo no solo debe requerir un aumento en la cantidad total de capital, sino también la racionalidad de la estructura de capital, entre natural y manufacturado, no debiendo traspasar los umbrales ecológicos del primero.

Juntamente con ello aparecieron varias aproximaciones hacia diversos conceptos que procuraban la posibilidad de tener un sistema de producción más acorde con los límites de la naturaleza. Estos esfuerzos fueron realizados desde diversas aproximaciones, por lo que mencionaremos algunas de ellas. Un concepto utilizado fue el de "metabolismo industrial" que según Anderberg (1998) fue propuesto Robert U. Ayres en 1988, quien señalaba que este podía verse como el conjunto integrado de procesos físicos que convierten las materias primas y la energía, además de la mano de obra, en productos terminados y desechos; siendo que el objetivo de los estudios del metabolismo industrial es tener un mejor conocimiento y comprensión de los usos sociales de los recursos naturales y su impacto total en el ambiente. 
Un concepto similar es el de simbiosis industrial que Neves et al (2020) afirman que tiene su génesis en la biología en la que la simbiosis representa la asociación de individuos de diferentes especies en una relación donde existe un beneficio mutuo. Según ellos, esta definición se ha transpuesto a industrias en las que la simbiosis industrial involucra a entidades tradicionalmente separadas en un enfoque colectivo de ventaja competitiva que implica el intercambio físico de materiales, energía, agua y subproductos.

Además, y tal vez la más mencionada es la ecología industrial, que en palabras de Erkman (1997) explora el supuesto que el sistema industrial puede verse como un cierto tipo de ecosistema dado que al igual que los ecosistemas naturales, los sistemas industriales pueden describirse como una distribución particular de materiales, energía y flujos de información, agregando además que todo el sistema industrial depende de los recursos y servicios proporcionados por la biosfera, de la cual no se puede disociar. Por su parte Jensen et al (2011) señalan que esta ha sido una fuente conceptual de esperanza para el desarrollo industrial sostenible durante al menos dos décadas, y podría decirse que la publicación del artículo de Frosch y Gallopoulos titulado "Strategies for Manufacturing" marcó el comienzo de un esfuerzo para diseñar los procesos industriales según lo observado dentro de la biosfera. Ellos señalan que el supuesto es que modelando los sistemas industriales en ecosistemas biológicos armoniosos y aparentemente eficientes en el uso de los recursos, es posible reorganizar los actuales sistemas industriales para que funcionen de una forma más benigna y ecológica para el ambiente.

Entonces, lo que es posible ver en todas estas aproximaciones es el mirar el sistema de producción como haciéndolos similar a los procesos naturales, o intentando hacerlos. Esto puede parecer más deseable que una propuesta concreta, dado que el funcionamiento de los procesos naturales es en general concatenados y establecidos en un orden determinado lo cual tiene otra lógica de los procesos industriales donde la prelación entre las acciones no siempre es una condición para que pueda ser llevada a cabo. Una crítica importante de estas aproximaciones viene de Ayres (2004) que argumentaba que un sistema económico no puede imitar el sistema ecológico debido a cuatro diferencias principales: (1) el sistema económico no tiene un productor primario que imite a los fotosintetizadores; (2) la salida es una mezcla heterogénea de diferentes productos y servicios, mientras que las únicas salidas de la biosfera incluyen desechos y materia muerta; (3) el concepto de mercado, trabajo y dinero no existe en la biosfera porque los intercambios son involuntarios; y (4) la evolución en el sistema económico está impulsada por innovaciones y nuevos descubrimientos, mientras que en la biosfera la evolución depende de las mutaciones aleatorias del genoma.

Este esfuerzo de juntar la economía con la naturaleza tiene una buena aproximación en la bioeconomía que según Mohammadian (2003) señala que esta tiene como objetivo de la bioeconomía lograr la síntesis de la biología y la economía, cerrar la brecha entre ambas ciencias y así acabar con la desunión y separación entre ellas. Según el autor la bioeconomía es un cambio paradigmático en la evolución de las disciplinas que investigan los problemas derivados del impacto de la actividad socioeconómica en el ambiente, los cuales no se deben únicamente a causas biológicas ni a causas económicas, sino a la interacción entre ellos, es decir son bioeconómicos. 
Sin embargo, este esfuerzo de construcción aun se encuentra en evolución y en donde más se ha avanzado se refiere a tópicos relacionados con la economía pesquera y la forestal, estando aun estos circunscritos a situaciones donde los bienes analizados tienen un precio de mercado y la discusión se basa en cuál debe ser la tasa de explotación óptima del recurso estudiado.

Definiendo la economia circular. Es en medio de estas propuestas que aparece la EC. Existen varias definiciones de lo que significa las mismas que se han planteado en diferentes ámbitos, desde el académico hasta los implementadores del concepto. Kirchherr et al (2017) hacen una evaluación de 114 definiciones de lo que significa EC, lo que a todas luces gráfica los diversos entendimientos que se tiene sobre este concepto. Ellos señalan que diversos autores sostiene que la definición más elaborada es la propuesta por Ellen MacArthur Foundation que dice que la EC es un sistema industrial que es restaurador o regenerativo por intención y diseño, reemplazando el concepto de "fin de vida" por restauración, cambiando hacia el uso de energía renovable, eliminando el uso de productos químicos tóxicos, que perjudican la reutilización, y que tiene como objetivo la eliminación de residuos mediante un diseño superior de materia, productos, sistemas $\mathrm{y}$, dentro de estos, un modelos de negocio. En términos concretos se propone modelos menos intensivos en el uso de materia y de energía concatenando procesos entre ellos.

Por su parte Korhonen et al (2018) proponen que la EC es una economía construida sobre sistemas de producción-consumo que maximizan el servicio producido a partir del flujo lineal naturaleza-sociedad-naturaleza materia y energía, la cual se elabora mediante el uso de flujos de materia cíclicos, fuentes de energía renovables y flujos de energía de tipo cascada. Según los autores el éxito de la EC contribuye a las tres dimensiones del desarrollo sostenible, dado que esta limita el flujo de producción a un nivel que la naturaleza tolera y utiliza los ciclos de los ecosistemas dentro de los ciclos económicos respetando sus tasas de reproducción naturales.

Al-Thani y Al-Ansari (2021) señalan que recientemente conceptos como la EC, la ecología industrial y el nexo entre energía, agua y alimentos han surgido como conceptos populares de manejo de recursos aplicados para promover el desarrollo sostenible y la eficiencia en su uso de recursos en varios sectores y estudios de casos. Ellos señalan que, en ausencia de una definición estandarizada de EC, muchos estudios han intentado desarrollar una definición científica formal. Los intentos de definirla han aludido a i) la circulación de recursos como un medio para promover el uso eficiente de materiales y energía, es decir, a través de la minimización de residuos y la recuperación de recursos, ii) utilización de fuentes de energía renovables y iii) adopción de un enfoque de diversificación, pensamiento sistémico y multinivel para construir sistemas resilientes. Los autores sostienen que la principal diferencia entre la EC y los otros conceptos es que esta es un sistema restaurativo, mientras que los otros conceptos son sistemas preventivos; donde los sistemas restauradores se refieren a un sistema resistente que puede volver a su forma inicial o que la materia y la energía se restablecen dentro del sistema nuevamente, mientras que los sistemas preventivos son aquellos que adoptan determinadas medidas para minimizar las pérdidas de energía y materia u optimizarlas dentro del sistema.

${ }^{1}$ Los autores utilizan este concepto en vez de hablar de ciclos (o reciclaje) de la energía porque esta no puede ser reciclada. 
En Iniciativa Financiera del PNUMA (2020) se menciona que: "Los comportamientos económicos más comúnmente asociados con el enfoque de la circularidad se describen a través del concepto 9-R que sirve de sustituto a una definición de la economía circular. Así mismo se señala que: "Estos comportamientos fueron originalmente acuñados bajo el concepto 3-R (reducir, reutilizar, reciclar), que luego fue desarrollado para transformarse en el concepto 6-R (con la adición de recuperar, rediseñar y refabricar) y más tarde evolucionó hacia el concepto 9-R (con la adición de reacondicionar, reparar y rechazar)".

Morseletto (2020) por su vez sostiene que la economía circular (CE) se puede definir como un modelo económico dirigido al uso eficiente de los recursos a través de la minimización de residuos, la retención de valor a largo plazo, la reducción de recursos primarios y ciclos cerrados de productos, partes de productos y materiales dentro de los límites de la normativa medioambiental, protección y beneficios socioeconómicos. Una EC tiene el potencial de conducir al desarrollo sostenible, mientras que desvincula el crecimiento económico de las consecuencias negativas del agotamiento de los recursos y la degradación ambiental.

Aún cuando existen diversas definiciones de EC lo que queda evidente en todos los conceptos es la búsqueda de un sistema productivo menos intensivo en materias y energía en sus dos formas, como bienes y servicios a ser usados por la sociedad y como residuos (males) de los procesos de procesos de producción y consumo. Aun cuando en las definiciones revisadas no se menciona la materia y la energía es importante hacer esta consideración porque ella nos alerta sobre las limitaciones físicas que tienen los procesos antropogénicos y que son una limitante que debe estar siempre presente en los análisis sobre los análisis de sostenibilidad, especialmente porque se relaciona con las leyes de la termodinámica.

Por ejemplo, Figge et al (2021) demostraron que un aumento en la circularidad de los recursos no significa necesariamente que estos se utilizarán de manera más eficiente, es decir la combinación de usuarios de recursos que minimiza el uso de recursos no es necesariamente la combinación que maximiza la ecoeficiencia. Para ellos la idea de que la economía circular da como resultado una ecoeficiencia infinita en el uso de recursos solo es válida en el improbable caso de circularidad perfecta, lo que apunta a la importancia de distinguir entre el concepto teórico de circularidad perfecta y el caso práctico de un uso circular imperfecto de los recursos. Esto es importante en la medida de que la circularidad tiene un límite propio en el objetivo que pretende alcanzar, dado que se tiene que pensar en una coherencia de producción industrial que puede tener alcances físicos de conseguirse, pero se depara con las limitaciones individuales del comportamiento maximizador de los productores. Es un problema parecido con la diferencia entre las soluciones individuales y la solución social que no siempre coinciden por lo que hay que ver cómo se corrige esa falla.

Economizando la economía circular:Es interesante como todos los conceptos sobre economía circular básicamente se habla de sobre cómo se debería de producir y cuáles serían las condiciones para hacerlo de tal forma que ello implique un menor impacto al ambiente. Sin embargo, hay propuestas limitadas sobre cómo todos estos conceptos deben ser considerados desde el punto de vista económico, especialmente 
si uno espera que por el nombre mismo exista toda una consideración económica por detrás de ello. En este sentido, Stahel (2016) señala que los modelos de negocios basados en la EC se dividen en dos grupos: los que fomentan la reutilización y prolongan la vida útil mediante reparaciones, refabricaciones, actualizaciones y modernizaciones; y aquellos que convierten los productos viejos en recursos nuevos mediante el reciclaje de los materiales. Lo evidente parece ser que la racionalidad que tienen los productores es la misma que aquella que señala la teoría económica siendo la única diferencia el espacio del negocio que se ocupa.

Para entender cómo la EC se puede encajar dentro de la lógica económica hay que establecer cuáles son las relaciones que se espera se generan entre ellas para a partir de esto relacionarlo con el funcionamiento económico. En primer lugar, es importante dejar en claro que los dos principios sobre los cuales la economía se ha construido son: la escasez relativa y la racionalidad. La escasez relativa se basa en el concepto que los bienes y servicios son relativamente escasos en la medida que, existiendo demanda para ellos, los productores estarán dispuestos producirlos. Esta escasez relativa se expresa mediante el sistema de precios. En el caso de la racionalidad esta se refiere a que los agentes económicos siempre procuran lo mejor para sí, ya sea en su papel de consumidor o de productor. Existe toda una discusión en la economía sobre el principio de la escasez, mencionando que esta puede ser absoluta en la medida que hay principios físicos que la fundamentan, pero ello no altera la lógica de lo que se plantea.

Basado en ello es importante conocer cómo es el flujo económico, que en términos sencillos tiene esta lógica.

\section{Figura 1}

\section{El proceso productivo simple}

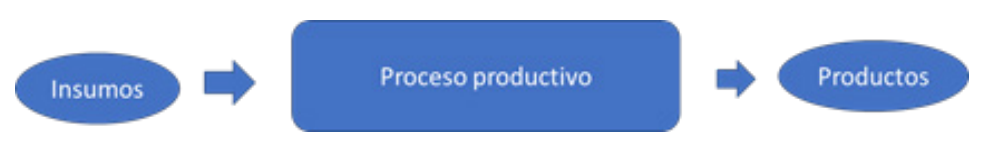

El proceso productivo es aquel donde un conjunto de insumos entra en él y después de una cierta combinación se generan productos, o, mejor dicho, bienes y males, los mismos que pueden ser expresados a su vez en materia y energía. Por el principio físico de la conservación, la cantidad de materia y energía que ingresa al proceso es la misma que sale de él. Así, cuando se habla de los resultados del proceso productivo, los productos son los bienes y los males que se generan, siendo que estos últimos son obtenidos no intencionalmente en el proceso, dado que estos no son completos y aparecen como residuos. Esta lógica es igualmente aplicable si la referencia fuese sobre procesos relacionados con el consumo.

En la medida que los productos que salen del proceso productivo pueden ser intermedios o finales; si son intermedios entrarán a un nuevo proceso productivo y si son finales, no. Los males, tienden generalmente a no continuar en el siguiente proceso, 
aunque también dependerá de cada caso específico, porque puede haber males que al final se conviertan en insumos para otros procesos. Un ejemplo claro es el de las aguas residuales, que siendo estas un producto no deseado, mal, del uso del agua potable, dependiendo de sus condiciones, puede ser usado en otros procesos.

Por otra parte, los insumos pueden ser elementos no generados por los humanos y otros que vienen de otros procesos productivos. Siendo esta una mejor representación del proceso económico sería:

\section{Figura 2}

Proceso Productivo completo

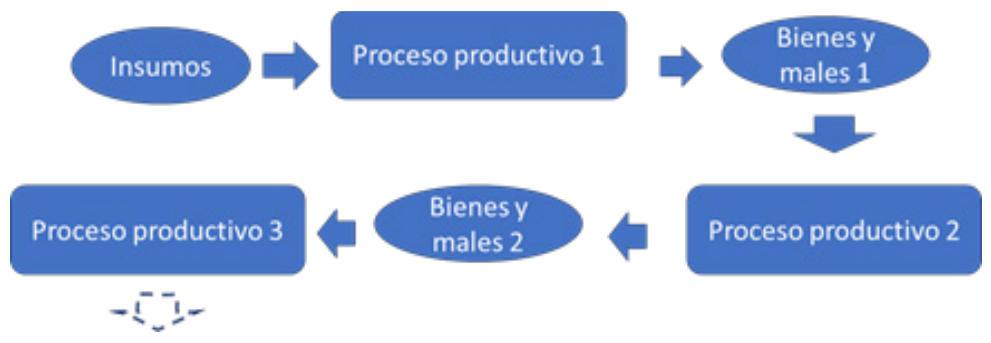

De esta forma, se puede decir que el actual proceso económico es un proceso no es necesariamente lineal, pero sí que tiene una sola dirección y que hay procesos que se complementan entre sí. El caso de las frutas envasadas es un buen ejemplo. Dentro del proceso de producción agrícola se obtiene como resultado de un conjunto de insumos (suelo, agua, semilla, abonos, etc.) la fruta y los residuos que se generan de ella (agua no incorporada en el producto, etc.). Este producto a su vez se constituye en un insumo si estuviésemos hablando de la industria de conservas, donde la fruta es transformada y se obtiene un producto diferente.

\section{Figura 3}

\section{Las 5 fases}

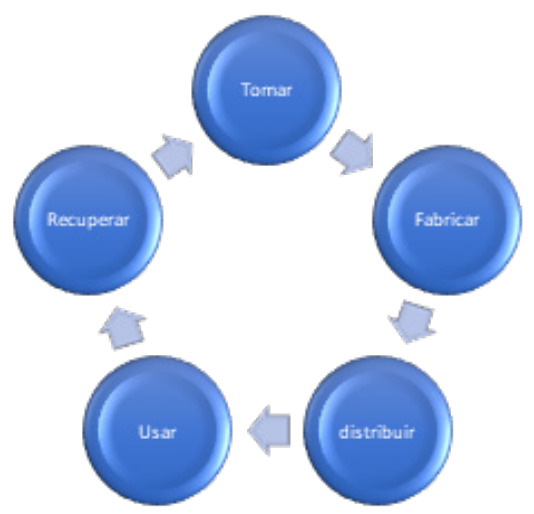

Saha et al (2021) proponen una visión ampliada del proceso productivo donde señalan que "tomar, fabricar, distribuir, usar y recuperar" son las cinco funciones que pueden reducir el uso de materias primas y estimular la reutilización y reciclaje de los 
productos.

Según los autores las empresas consumen materias primas durante la función de tomar y las convierten en productos terminados, fabricar; distribuir se relaciona con poner los productos terminados a disposición de los usuarios; mientras que el uso permite a los consumidores obtener beneficios de la utilidad de los productos, siendo que la recuperación gestiona el estado de final de vida útil del producto mediante la reutilización y el reciclaje. Esta aproximación de Saha et al (2021) lo que hace es complementar el proceso productivo con los procesos de realización del producto. Lo interesante en este caso es que se ve el proceso económico en su conjunto donde en realidad se van produciendo las mismas consideraciones que se señalaron anteriormente como un proceso concatenado como en realidad sucede.

En esta lógica el sistema económico y su contrapartida física tienen una relación estrecha. A cada paso de un bien por el sistema económico hay una contrapartida monetaria que justamente obedece a la dinámica de los procesos económicos, donde los bienes son demandados por los consumidores y por ello producidos por los productores. Esta correspondencia física-monetaria no es necesariamente equivalente porque existen bienes y servicios que no son escasos por lo que no hay una necesidad de parte de la sociedad de expresar esta falta de escasez. Por ejemplo, la luz solar, ella hace parte del proceso de producción agrícola y permite que se generen bienes y servicios, pero al no ser escasos no son considerados en el sistema económico. Sin embargo, puede haber bienes que sean escasos y que sin embargo no tienen precios que expresen esta escasez, que es lo que se conoce como fallas de mercado, donde dada alguna condición relacionada con la ausencia de los principios de la formación de precios, estos no se forman, entre los cuales pueden estar la exclusividad, rivalidad, derechos de propiedad, información imperfecta, entre otros. Hay una extensiva explicación en la economía de cómo corregir esta situación.

Entretanto, esta lógica no es la misma con la que opera los balances de materia y energía que se encuentran dentro del concepto de economía circular donde la idea de estos es justamente minimizar el uso de mismos, así como su reducción después de los procesos de transformación. Un poco para entender sus fundamentos es necesario que se entienda cómo operaría la EC. En este sentido lo que debe suceder en los procesos productivos y de consumo es que deben diseñarse pensando en el límite dado por la cantidad de materia y de energía que se dispone; que sea posible reemplazar insumos por otros que sean menos demandantes de materia y energía; que sean generadores de menor cantidad de residuos en todas sus formas, y que sea posible que residuos de un proceso puedan volver a entrar al mismo proceso o hacia otro proceso.

Para que ello sea posible es necesario ver cómo estas consideraciones se insertan dentro del proceso económico lo que significa que estos deben de apoyarse dentro de la racionalidad económica. Así, se puede decir que la EC requiere que se cambien los insumos actuales por nuevos insumos, digamos menos demandantes de materia y energía. Suponiendo que los productores son racionales, es decir maximizadores del beneficio, esto solo sucederá por la orientación que los precios den. Si ellos siguen con su lógica entonces solo se verán incentivados a cambiar de insumos si los equivalentes son menos onerosos que los actuales en las mismas condiciones. Es decir, teniendo la 
siguiente función de beneficios, donde p es el precio del bien final, $Q$ el bien final, y $m$ y elos insumos y los $r_{\mathrm{i}}$ sus respectivos precios, por lo que dado:

$$
\text { (1) } Q=\left(m_{i}, e_{i}\right)
$$

El productor maximiza (2)

(2) $\pi_{1}=p_{1} Q-r_{1} m_{1}-r_{2} e_{1}$

Entonces para que los productores puedan reemplazar sus actuales insumos por otros menos intensivos en materia y energía deberá haber precios de los insumos que en la suma sean menores que los actuales, $\mathrm{r}_{3} \mathrm{y}_{4}$, es decir:

$$
\text { (3) } \quad r_{1} m_{1}+r_{2} e_{1} \geq r_{3} m_{2}+r_{4} e_{2}
$$

Para que la misma entre dentro de la lógica de la EC debe suceder también que:

$$
\text { (4) } \quad m_{1} \geq m_{2} y \quad e_{1} \geq e_{2}
$$

Sin esa restricción puede suceder que, en la maximización del beneficio, la reducción de alguno de los precios de un insumo, materia o energía compense que se pueda aumentar el uso del otro. Si eso sucediese esta sería una condición normal y no tendría efecto sobre la disponibilidad de materia y energía. Con lo que el productor decidirá utilizar estos insumos con menos impacto en el ambiente. Claro para que ello sea posible deberá haber alguna intervención en el mercado, porque suponiendo que, existiendo competencia perfecta, es decir con productores informados, no han elegido esos insumos porque al final sus costos se harían mayores y reducirían sus beneficios.

Sin embargo, otra alternativa para mantener al menos el mismo nivel de beneficio es que aun si la relación (3) fuese inversa esta pueda ser adoptada a través de una compensación en el nivel de precios recibido. Es decir, tener un $\mathrm{p}_{2}$ mayor que $\mathrm{p}_{1}$ que pueda compensar el incremento en los costos de tal manera que los beneficios se mantengan al menos constantes.

$$
r_{3} m_{2}+r_{4} e_{2} \geq r_{1} m_{1}+r_{2} e_{1}
$$

Entonces deberá suceder:

$$
\text { (6) } \pi_{1}=p_{2} Q-r_{3} m_{2}+r_{4} e_{2}
$$


De tal forma que el nuevo precio $\mathrm{p}_{2}$ compensa el incremento en los costos $\mathrm{y}$ mantiene al menos los beneficios en el mismo nivel que el anterior. Por eso la restricción (4) es importante porque al final lo que se desea es que la presión sobre el uso de materia y energía disminuya. Esto es totalmente aplicable a la introducción de paquetes tecnológicos mejores que permitan producciones mayores por la misma cantidad de materia o energía o que a menores niveles de materia y energía se pueda obtener el mismo nivel de producto.

Pero si ello no ha sucedido hasta el momento es que los precios actuales $\left(\mathrm{r}_{1}, \mathrm{r}_{2}\right)$ han orientado a un uso de un paquete tecnológico determinado $\left(\mathrm{m}_{1}, \mathrm{e}_{1}\right)$ y no han permitido el uso del paquete tecnológico deseado $\left(\mathrm{m}_{2}, \mathrm{e}_{2}\right)$. Entonces, para que el cambio efectivamente suceda se tiene que cambiar esa relación de precios. La idea es que se puede penalizar los precios actuales por justamente una especie de costo de oportunidad del agotamiento que estaría relacionado con la indisponibilidad futura del insumo, o por un subsidio que justamente reconozca esa diferencia mencionada.

Sin embargo, podría dejarse verse esta situación desde otra óptica. Es decir, dado que no se ha optado por un nuevo paquete tecnológico $\left(\mathrm{m}_{2}, \mathrm{e}_{2}\right)$ porque los precios de sus insumos $\left(\mathrm{r}_{3}, \mathrm{r}_{4}\right)$ son más caros, estando por lo tanto negada la posibilidad de que los productores decidan asumir el cambio sin tener un beneficio evidente por esa modificación. Aquí se podría justamente promover los cambios a través del precio final del bien o servicio a ser producido, teniendo un $\mathrm{p}_{2} \geq \mathrm{p}_{1}$ que justamente permita pasar a una forma de producción diferente que sea menos impactante al ambiente. La elección de ser un subsidio o un incremento en el precio dependerá de las opciones de política que se dispongan.

Es necesario considerar que los precios no cambian de un momento a otro y tampoco lo hacen los empresarios. Eso significa que para que el proceso sea efectivamente viable, alguien, el gobierno, por ejemplo, debería de financiar las acciones que demanda la economía circular, que no únicamente sería la alteración de la relación de precios sino también los procesos tecnológicos suficientes para ello. Es por ello por lo que para hacerla viable es necesario que se puedan dar una serie de condiciones.

1. Que existan precios que orienten la utilización de insumos y productos con el menor contenido de materia y energía. Para que los precios puedan cambiar existen al menos dos formas de poder hacerlo. Uno sería con la intervención gubernamental que artificialmente altere los precios con diversos mecanismos que existen a disposición y la otra es un cambio en los demandantes, que prefieran productos con características de menor impacto al ambiente. Para que ello sea posible estos tendrían que cambiar sus preferencias y eso no sucede en el corto plazo.

2. Para que las acciones tengan fehacientemente una orientación en los precios, deben cambiar las preferencias de los consumidores. Aquí el problema es que este cambio no sucede en el corto plazo y va a depender de que se puedan hacer campañas de convencimiento dirigidos hacia los consumidores de tal forma que se replanteen sus consumos actuales por otros con productos con características menos 
impactantes. Esta situación solo debe suceder al menos en el mediano plazo por lo que es necesario que se generen elementos de intervención económica que ayuden a cambiar los comportamientos de las personas.

3. Este esfuerzo no puede ser una acción aislada de un grupo de personas o de un país en forma individual. No olvidemos que los esfuerzos para cambiar de una forma de producir a otro implican pérdida de competitividad y justamente para que el proceso pueda seguir existiendo es importante que todos los países puedan actuar de forma conjunta, sino los esfuerzos serán aislados y sin repercutir efectivamente en la sociedad. Como sostiene Figge et al (2021) si bien una sola empresa puede adoptar sistemas para utilizar sus recursos de forma circular, los sistemas de economía circular también pueden abarcar varias empresas diferentes, como las que se observan en la simbiosis industrial. Por lo que, al formar grupos de colaboración, dentro de los cuales los recursos fluyen de un lado a otro entre los usuarios de los recursos, estos grupos necesitan menos recursos. En este contexto, los autores sostienen que existe un paralelo entre el efecto de la diversificación en las finanzas y el efecto de reducción de recursos de los sistemas de economía circular en general y la simbiosis industrial en particular: los riesgos se reducen en contextos financieros mediante la construcción de una cartera de activos; el uso de recursos naturales se reduce en la economía circular mediante la creación de una cartera de usuarios de recursos que colaboran entre sí.

Como se señala en el último punto es importante que se pueda considerar a la EC dentro de un contexto mayor, pero ello implica que pueda existir un sistema de precios que pueda ayudar a considerar todos sus elementos. Es claramente esta una tarea difícil, aunque ya varios autores han intentado avanzar en este tema. Bouman et al (2000) aplican tres modelos: análisis de flujo de sustancias, evaluación del ciclo de vida y análisis de equilibrio económico parcial señalando que existe una amplia gama de otros modelos, pero aseguran que estos son representativos de las diferencias típicas que existen entre los distintos tipos de modelos.

Por su parte Patterson (2002) revisa la teoría y el método de fijación de precios ecológicos y lo aplica a la valoración de los procesos y servicios de la biosfera señalando que los precios ecológicos valoran los procesos de la biosfera, sobre la base de las interdependencias biofísicas entre todas las partes del ecosistema, no solo aquellas que tienen un valor directo u obvio para los humanos. El autor señala también una de las principales críticas hacia esta aproximación señalando que la diferencia clave entre "precios ecológicos" y "precios de mercado" es que los primeros miden el valor en términos de las interdependencias biofísicas en el sistema; mientras que los últimos se basan en las preferencias de los consumidores y otros factores que determinan el valor de cambio en los mercados.

¿Es posible la implementación de la EC? Diversos autores han señalado los problemas para implementar la EC. Por ejemplo, Galvão et al (2018) señalan que, entre las barreras identificadas en la literatura, las tecnológicas, políticas y regulatorias, financieras y económicas, gerenciales, indicadores de desempeño, clientes individuales y sociales son las más frecuentes, representando los principales desafíos para la implementación de la CE. También se identificaron las barreras para las EC en los parques ecológicos que los autores dividen en dos categorías: 
(1) externas (barrera de apoyo al capital, a las políticas y de acceso a la información) e (ii) internas (recursos tangibles, intangibles, capacidades).

Por su parte Stumpf et al (2021) sugieren que las empresas enfrentan varios desafíos al implementar la EC, donde la falta de definiciones y estándares, la falta de aplicación y cooperación del gobierno y las barreras técnicas han sido las más importantes. Mencionan que en un análisis empírico intersectorial se encontró que la complejidad de los procedimientos legales y los costos relacionados con el cumplimiento de las regulaciones son las barreras más significativas. Asimismo, en su revisión de otros estudios mencionan que estos han encontrado que las barreras culturales (por ejemplo, la falta de interés y conciencia del consumidor y una cultura organizacional vacilante) seguidas de las barreras del mercado (por ejemplo, precios bajos de materiales vírgenes, altos costos de inversión iniciales) son las barreras más apremiantes para una EC. Además, los autores mencionan que los obstáculos normativos que prevalecen en los proyectos deben abordarse de forma más directa, por ejemplo, mediante la imposición de cuotas y objetivos sólidos. Estos deben priorizar varios principios de la EC al mismo tiempo que equilibran de manera sensata las compensaciones asociadas. Sin embargo, estas consideraciones deberían abordarse con cautela dado que puede dar paso a comportamientos oportunistas, como, por ejemplo, un objetivo de reciclaje elevado podría promover el reciclaje a costa de reducir y reutilizar, que son de hecho las opciones de EC más deseables.

Según Saha et al 2021, tiene que existir un marco para la implementación de la EC que se puede resumir en la tabla a seguir.

\section{Figura 4}

Puntos de acción para la implementación de la EC
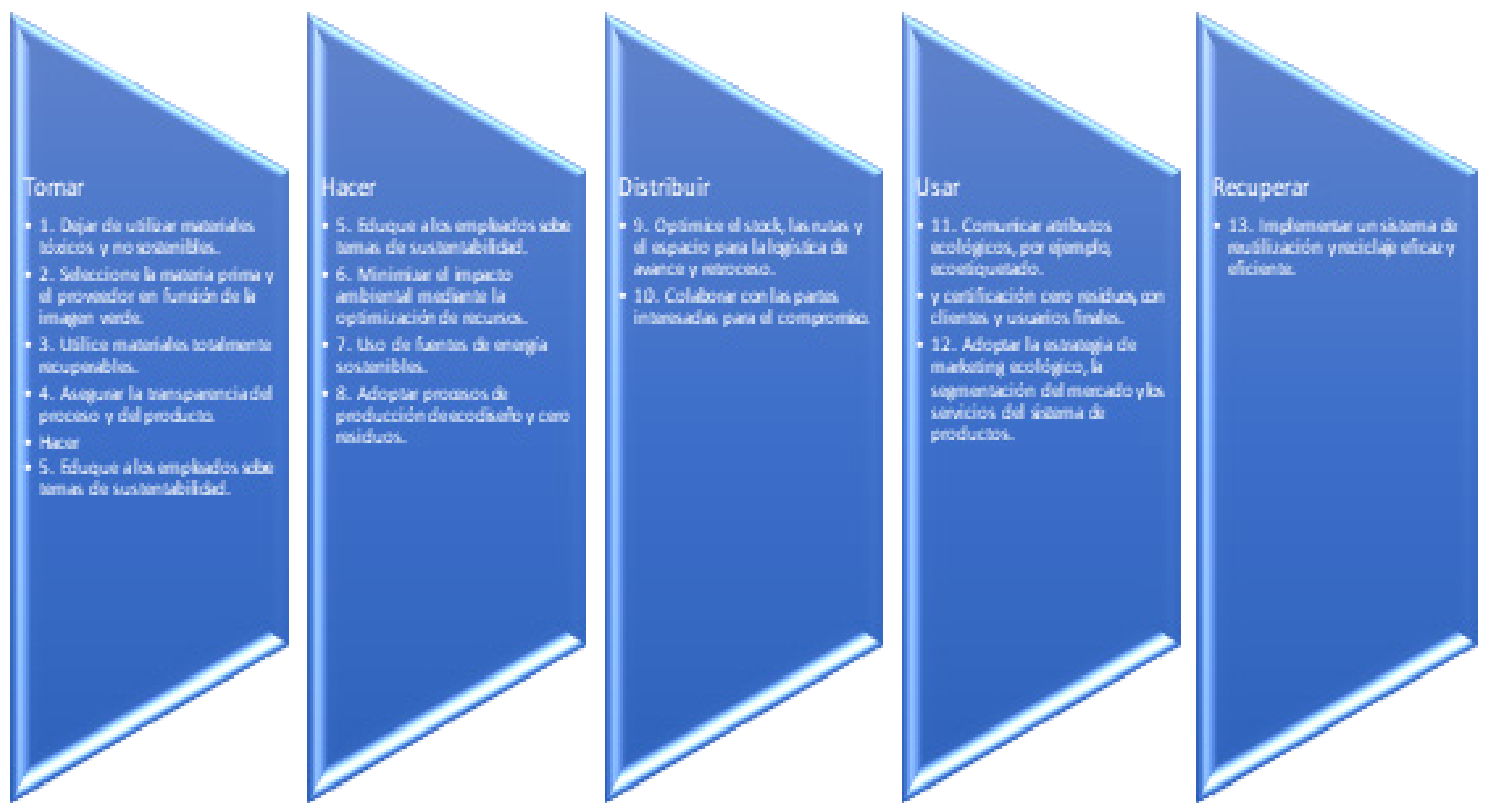
Según ellos la implementación exitosa de EC depende de varios factores internos y externos. Los factores externos incluyen la política pública, las condiciones del mercado, el desarrollo tecnológico y las partes interesadas, mientras que los factores internos son los recursos, las capacidades y las competencias de la empresa.

\section{Conclusión}

Una de las consideraciones más importantes es que la EC es parte de un esfuerzo que ya se venía haciendo desde hace algún tiempo especialmente por reconocer de los límites que tiene la naturaleza de proveedor de insumos y de asimilador de residuos. La diferencia es que algunas propuestas sobre la asimilación del comportamiento productivo con la lógica de los seres vivos se encontraron con serios límites en su operabilidad. Ya la EC reconoce la existencia de la circularidad y que esa circularidad tiene un límite físico en si propio, pero justamente puede ser una alternativa que permita una menor intensidad en el uso de materiales y energía intentando que en la medida de lo posible se pueda promover la circularidad dentro de los procesos productivos.

Lo que si llama la atención en la discusión del concepto es la falta de fundamento económico por detrás del mismo. Es decir, la mayor parte de las propuestas señalan las consideraciones físico-productivas que deben de generarse sin hacer mención del principio maximizador de los beneficios que los productores tienen. Proponer políticas sin esa consideración solo van a permitir que se puedan dar soluciones incompletas, donde uno de los elementos del cambio escapa a esa consideración que justamente está relacionado con la lógica del agente que es lo que se pretende cambiar. Y claro, esa falta de argumentos económicos lleva a que cuando se incluye alguna política relacionada con el impulso de la EC se realice considerando el apoyo gubernamental, lo cual es una herramienta de corto plazo y por lo tanto poco sostenible. El eje básico de todo cambio debe estar en el mismo consumidor, que, mostrando sus preferencias por determinados tipos de comportamientos por parte del productor, este se verá reflejado en la estructura de precios, con lo que puede sostener sus actividades y efectivamente promover el cambio. La EC para que efectivamente pueda funcionar no se debe olvidar de la economía.

\section{Declaración de conflicto de intereses}

El autor declara no tener conflicto de intereses financieros ni personales que puedan influir inapropiadamente en el desarrollo de este articulo. 


\section{Referencias}

Al-Thani, N.A. \& Al-Ansari, T. (2021). Comparing the convergence and divergence within industrial ecology, circular economy, and the energy-water-food nexus based on resource management objectives. Sustainable Production and Consumption, 27, 1743-1761, ISSN 2352-5509, https://doi.org/10.10 16/j.spc.2021.04.008.

Anderberg, S. (1998). Industrial metabolism and the linkages between economics, ethics and the environment. Ecological Economics 24, 311-320.

Ayres, R. U. (2004). On the life cycle metaphor: where ecology and economics diverge. Ecological Economics, 48 (4), 425-438, ISSN 0921-8009, https://doi. org/10.1016/j.ecolecon.2003.10.018.

Bouman, M.; Heijungs, R.; van der Voet, E.; van den Bergh, J.C.J.M ; Huppes, G. (2000). Material flows and economic models: an analytical comparison of SFA, LCA and partial equilibrium models. Ecological Economics, 32 (2), 195-216, ISSN 0921-8009, https://doi.org/10.1016/S0921-8009(99)00091-9.

Erkman, S. Industrial ecology: an historical view. (1997). Journal of Cleaner Production.. 5 ( 1-2), 1-10.

Figge, F; Thorpe, A. S.; \& Manzhynski, S. (2021). Between you and I: A portfolio theory of the circular economy. Ecological Economics, 190, 107190, ISSN 0921-8009, https://doi.org/10.1016/j.ecolecon.2021.107190.

Galvão, G.D.A.; Nadae, J. de; Clemente, D. H.; Chinen, G.; \& Carvalho, M. M. (2018). Circular Economy: Overview of Barriers. Procedia CIRP, 73, 79-85, ISSN 2212-8271, https://doi.org/10.1016/j.procir.2018.04.011.

Iniciativa Financiera del PNUMA. (2020). Financiando la circularidad: Desmitificando las finanzas para economías circulares.

Jensen, P.1 D.; Basson, L.; \& Leach, M. (2011). Reinterpreting Industrial Ecology. Journal of Industrial Ecology, 15(5), 680-692. doi:10.1111/j.1530-92 90.2011.00377.x

Kirchherr, J.; Reike, D.; \& Hekkert, M. (2017). Conceptualizing the circular economy: An analysis of 114 definitions. Resources, Conservation and Recycling, 127, 221-232, ISSN 0921-3449, https://doi.org/10.1016/j.resconrec.2017.09.005

Korhonen, J.; Honkasalo, A.; \& Seppälä, J. (2018). Circular Economy: The Concept and its Limitations. Ecological Economics, 143, 37-46, ISSN 0921-8009, https://doi.org/10.1016/j.ecolecon.2017.06.041 
Mohammadian M. (2003). What Is Bioeconomics: Biological Economics?. Journal of Interdisciplinary Economics. 14(4), 319-337. doi:10.1177/0260107 9X03001400401

Morseletto, P. (2020). Targets for a circular economy. Resources, Conservation and Recycling, 153, 104553, ISSN 0921-3449,_ https://doi.org/10.1016/j. resconrec.2019.104553.

Neves, A.; Godina, R.; Azevedo, S. G.; \& Matías, J. C.O. (2020). A comprehensive review of industrial symbiosis. Journal of Cleaner Production, 247, 119113, ISSN 0959-6526, https://doi.org/10.1016/j.jclepro.2019.119113.

Patterson, M. G. (2002). Ecological production based pricing of biosphere processes. Ecological Economics, 41, Issue 3, pp 457-478, ISSN 0921-8009, https://doi. org/10.1016/S0921-8009(02)00094-0

Saha, K., Dey, P. K., \& Papagiannaki, E. (2021). Implementing circular economy in the textile and clothing industry. Business Strategy and the Environment, 3a4), 1497-1530. doi:10.1002/bse.2670

Shi, L.; Han, L.; Yang, F.; Gao, L. (2019). "The Evolution of Sustainable Development Theory: Types, Goals, and Research Prospects" Sustainability 11(24), 7158. https://doi.org/10.3390/su11247158

Stahel, W. (2016). The circular economy. Nature 531, 435-438 https://doi.org/10. $\underline{1038 / 531435 \mathrm{a}}$

Stumpf, L.; Schöggl, J.; \& Baumgartner, R. J. (2021). Climbing up the circularity ladder? - A mixed-methods analysis of circular economy in business practice. Journal of Cleaner Production. 316, 128158, ISSN 0959-6526, https://doi.org/10.1016/j.jclepro.2021.128158.

United Nations Environment Programme - UNEP. (2021). Emissions Gap Report 2021: The Heat Is On - A World of Climate Promises Not Yet Delivered Executive Summary. Nairobi.

UN Environment (2019). Global Environment Outlook - GEO-6: Healthy Planet, Healthy People. Nairobi. DOI 10.1017/9781108627146. 